\title{
GABAergic Control of Action Potential Propagation along Axonal Branches of Mammalian Sensory Neurons
}

\author{
Dorly Verdier, ${ }^{1}$ James P. Lund, ${ }^{1,3}$ and Arlette Kolta ${ }^{1,2,3}$ \\ ${ }^{1}$ Centre de Recherche en Sciences Neurologiques, and ${ }^{2}$ Faculté de Médecine Dentaire, Université de Montréal, Montréal, Québec H3C 3J7, Canada, and \\ ${ }^{3}$ Faculty of Dentistry, McGill University, Montréal, Québec H3A 2B2, Canada
}

\begin{abstract}
The main axons of mammalian sensory neurons are usually viewed as passive transmitters of sensory information. However, the spindle afferents of jaw-closing muscles behave as if action potential traffic along their central axons is phasically regulated during rhythmic jaw movements. In this paper, we used brainstem slices containing the cell bodies, stem axons, and central axons of these sensory afferents to show that GABA applied to the descending central (caudal) axon often abolished antidromic action potentials that were elicited by electrical stimulation of the tract containing the caudal axons of the recorded cells. This effect of GABA was most often not associated with a change in membrane potential of the soma and was still present in a calcium-free medium. It was mimicked by local applications of muscimol on the axons and was blocked by bath applications of picrotoxin, suggesting activation of $\mathrm{GABA}_{\mathrm{A}}$ receptors located on the descending axon. Antidromic action potentials could also be blocked by electrical stimulation of local interneurons, and this effect was prevented by bath application of picrotoxin, suggesting that it results from the activation of $\mathrm{GABA}_{\mathrm{A}}$ receptors after the release of endogenous GABA. We suggest that blockage is caused mainly by shunting within the caudal axon and that motor command circuits use this mechanism to disconnect the rostral and caudal compartments of the central axon, which allows the two parts of the neuron to perform different functions during movement.
\end{abstract}

Key words: primary afferents; presynaptic inhibition; antidromic firing; mastication; central pattern generation; action potential block

\section{Introduction}

It is generally assumed that the axonal arbor of vertebrate neurons conducts action potentials without modulation over most of their length. In primary afferent neurons, spikes normally flow from the peripheral branch of the axon, in which they are generated toward the soma and central branch. However, in many of these neurons, modulation is known to take place at presynaptic terminals, in which GABA-induced depolarization inhibits local synaptic transmission (Cattaert et al., 1992, 1994; Gossard, 1996). It has been suggested that this process, termed primary afferent depolarization (PAD), may have distant effects. For instance, action potentials do not flow into all intraspinal collaterals of the same muscle afferent fiber during PAD (Eguibar et al., 1994, 1997; Lomeli et al., 1998), and strong PADs generate action potentials that flow back toward the soma and the periphery (Dubuc et al., 1985; Gossard et al., 1991; Cattaert et al., 1994). For clarity sake, we will refer to spikes generated in the periphery as orthodromic and those generated at the terminals of central branches as antidromic. The antidromic spikes can reach the peripheral axonal terminal, in which they modify the sensitivity of sensory end organs (Bevengut et al., 1997; Gossard et al., 1999), but they may also have a motor function.

Evidence of this comes from our recent work on primary af-

Received Sept. 9, 2002; revised Dec. 2, 2002; accepted Jan. 2, 2003.

This work was supported by the Canadian Institutes for Health Research.

Correspondence should be addressed to Dr. Arlette Kolta, Université de Montréal, Pavillon Paul Desmarais, C.P. 6128, Succursale Centre Ville, Montréal, Québec H3C 3J7, Canada. E-mail: arlette.kolta@umontreal.ca.

Copyright $\odot 2003$ Society for Neuroscience $\quad 0270-6474 / 03 / 232002-06 \$ 15.00 / 0$ ferents that innervate the spindles of jaw-closing muscles. In contrast to other primary afferents, their cell bodies are located in the trigeminal mesencephalic nucleus (NVmes) and not in a dorsal root ganglion. Furthermore, their somata receive synaptic inputs and are electrotonically coupled through gap junctions (Lazarov, 2002) (Fig. 1). During fictive mastication (fictive is the term for motor patterns generated under paralysis), the firing patterns recorded from the soma differ markedly from those recorded from the caudal compartment of the central axon of these afferents in rabbits (Westberg et al., 2000). In the majority (65\%) of extracellular recordings performed near the soma, activation of the motor circuits during fictive mastication does not alter tonic orthodromic activity induced by stretch of the jaw-closing muscles, and, in only one-third of cases, a phasic inhibition of this activity is observed, coincident with the jaw-opening phase (Fig. $1, J O)$ of the cycle. In contrast, phasic inhibition is seen in the great majority $(83 \%)$ of recordings from the caudal compartment of the central axon. In addition, this inhibition alternates with phasic excitation occurring in the jaw-closing phase (Fig. 1, $J C)$. The extra spikes appearing in the jaw-closing phase have been attributed to antidromic action potentials generated by PAD of the central terminals (Kolta et al., 1995). These spikes do not appear to reach the soma (Fig. 1), but, there is evidence, on the basis of spike-triggered averaging, that they do reach trigeminal motoneurons via collaterals (Westberg et al., 2000). These results suggest that an active mechanism (Fig. 1, A) splits the neuron into two functionally distinct compartments during mastication: a rostral compartment that includes the peripheral axon, 


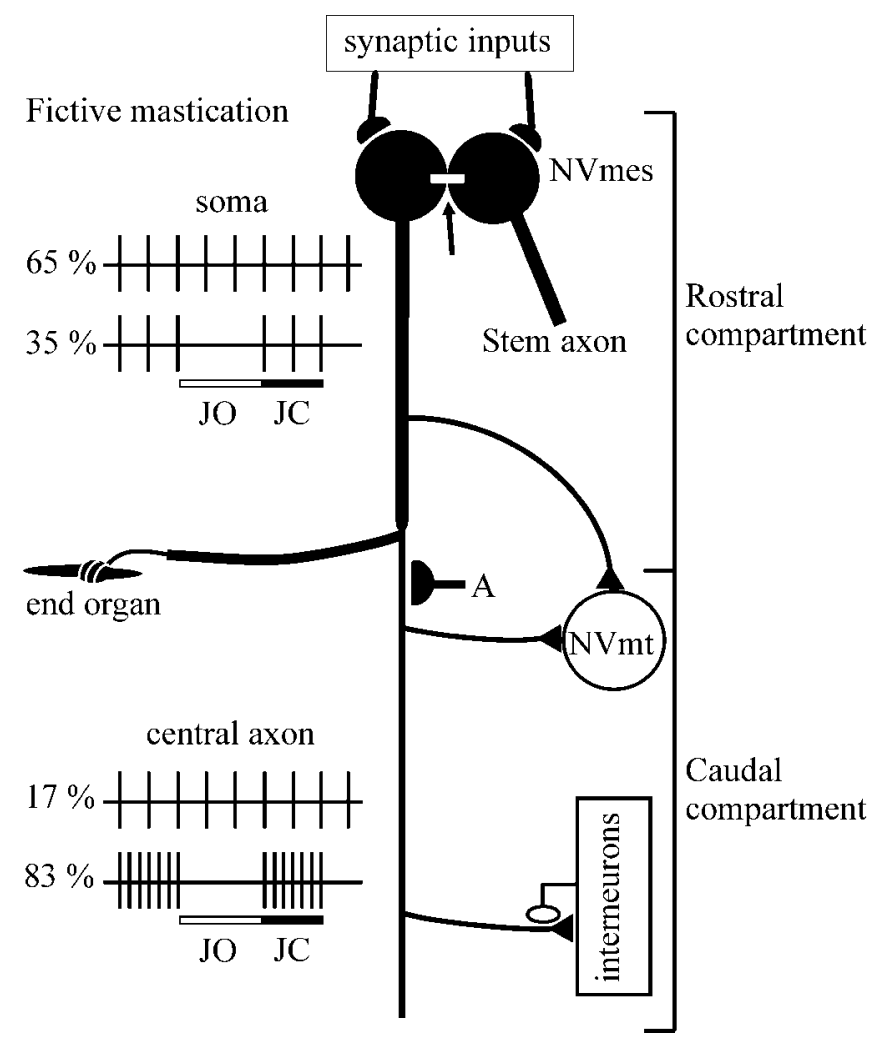

Figure 1. Functional compartmentalization of jaw-closing muscle spindle afferents (from Kolta et al., 1995; Westberg et al., 2000). During fictive mastication, stretch-induced tonic activity is unaltered in $65 \%$ of somatic recordings and is phasically inhibited during the jaw opening $(J 0)$ phase in $35 \%$ of cases. In contrast, phasic inhibition during the jaw-opening phase alternates with a phasic excitation during the jaw-closing $(J C)$ phase in the majority $(83 \%)$ of recordings from caudal axons. $A$, Hypothetical synapses on the axon that stop antidromic potentials from reaching the cell body. Arrow, Gap junction between somata.

soma, stem axon, and associated collaterals, and a caudal compartment formed by the descending central axon and its branches (Kolta et al., 1995; Westberg et al., 2000).

Here we test the hypothesis that the decoupling of the two parts of the axonal tree is controlled by GABAergic synapses on the axonal trunk.

\section{Materials and Methods}

We began by applying GABA to the axonal trunk to see whether this would prevent invasion of the cell body by action potentials generated in the caudal axon. We used an in vitro brainstem slice preparation that contains the somata and central axons of trigeminal muscle spindle afferents that were labeled by injections of $\mathrm{DiIC}_{18}(3)$ into the masseter muscles of rats at birth.

Preparation of slices. Figure $2 \mathrm{~A}$ shows a sagittal drawing of the brainstem with an outline of the cell body and the axonal branches of a trigeminal muscle spindle primary afferent neuron modified from Dessem and Taylor (1989). Their central axons form a descending tract that supplies the trigeminal motor nucleus (NVmt) and several groups of interneurons in the supratrigeminal and intertrigeminal areas (SupV and IntV, respectively) and in the spinal trigeminal nucleus (Dessem and Taylor, 1989; Luo and Li, 1991; Bae et al., 1996).

To visualize the cells from which to record, crystals of the carbocyanine dye DiI $C_{18}$ ([1,1'-dioctadecyl-3,3,3',3'-tetra-methylindocarbocyanine perchlorate]; Molecular Probes, Eugene, OR) were inserted in the masseter muscles of cryoanesthetized rat pups $(0-2 \mathrm{~d})$ and allowed to diffuse for 1-3 weeks before slice preparation. Nine- to 28-d-old Sprague Dawley rats (Charles River, Montreal, Quebec, Canada) were anesthetized by methoxyflurane inhalation (Metofane; Janssen Pharmaceuticals,
North York, Ontario, Canada) and decapitated. The brainstem was dissected, cooled in oxygenated ice-cold $\left(4^{\circ} \mathrm{C}\right)$ modified artificial CSF (aCSF) (in mm: 225 sucrose, $5 \mathrm{KCl}, 1.25 \mathrm{KH} 2 \mathrm{PO} 4,4 \mathrm{MgSO}_{4}, 0.2 \mathrm{CaCl} 2$, $20 \mathrm{NaHCO} 3$, and $10 \mathrm{D}$-glucose), and embedded in agar (Aghajanian and Rasmussen, 1989). The rostral end of the block was cut at $55^{\circ}$ from the horizontal axis. Sections ( $400-\mu \mathrm{m}$-thick), cut parallel to this plan using a vibratome, were transferred to an interface-type chamber saturated with a humidified mixture of $95 \% \mathrm{O}_{2}$ and $5 \% \mathrm{CO}_{2}$ and maintained at $29-$ $32^{\circ} \mathrm{C}$. They were perfused $(1 \mathrm{ml} / \mathrm{min})$ with modified aCSF for the first 20 min, then with a mixture $(50 \%-50 \%)$ of modified and normal aCSF (in mм: $125 \mathrm{NaCl}, 5 \mathrm{KCl}, 1.25 \mathrm{KH} 2 \mathrm{PO} 4,1.3 \mathrm{MgSO}_{4}, 2.4 \mathrm{CaCl}$, $26 \mathrm{NaHCO} 3$, and $25 \mathrm{D}$-glucose) for another $20 \mathrm{~min}$, and then with normal aCSF for the rest of the experiment. The slices were visualized at low magnification $(5 \times)$ under epifluorescence, and the trigeminal mesencephalic and motor nuclei were identified using a rhodamine filter. All procedures for dye injections and slice preparation conformed with national ethics committee guidelines and were approved by an institutional ethics committee.

Electrophysiology. The slices were viewed with an epifluorescence microscope (Nikon, Tokyo, Japan). Figure $2 B$ shows the labeling of trigeminal afferents (NVmes) and motoneurons (NVmt), as well as facial motoneurons (NVII) obtained by the dye injections. DiI-labeled cell bodies of masseteric spindle afferents in NVmes were targeted for recording with glass microelectrodes (80-200 M $\Omega$ ) filled with $2 \%$ Neurobiotin dissolved in potassium acetate ( 1 and $3 \mathrm{M}$ ). The recording $(R)$ and stimulating $(S)$ positions are indicated on the schematic drawing of the slice in Figure $2 \mathrm{~B}$. A diagrammatic neuron is drawn in red to help visualize how these relate to the axonal course. Data were recorded through a bridge circuit with an Axoclamp 2B amplifier (Axon Instruments, Foster City, CA) and sampled at $20 \mathrm{kHz}$, except when monitoring the effects of GABA over long periods. In these cases, the sampling rate varied from 1 to $10 \mathrm{kHz}$. Data were stored on disk and analyzed using pClamp 6-8 software (Axon Instruments). Antidromic action potentials were evoked by electrically stimulating the descending central axonal tract through bipolar twisted nichrome wire electrodes ( $25 \mu \mathrm{m}$ diameter). The intensity and duration $(0.05-0.3 \mathrm{msec})$ of pulses were gradually increased to establish the threshold of antidromic spikes, and stimulus intensity was set at twice threshold (50 $\mu \mathrm{A}$ to $10 \mathrm{~mA}$ ). High-frequency following (100$250 \mathrm{~Hz}$ ) was used to ensure that the elicited spikes resulted from direct activation of the neuron. Spike onset latencies and amplitudes were measured. All quantitative data are expressed as mean \pm SE. Differences between measures were compared with Student's paired $t$ test (SigmaStat software; SPSS, Chicago, IL) and were considered to be significant if the probability of $\alpha$-type error was $<0.05$. Correlations between variables were established with Pearson's correlation test and linear regression.

Drug application. GABA $(1,10$, and $50 \mathrm{~mm})$ and muscimol $(50 \mu \mathrm{M})$ were dissolved in Fast Green-containing normal aCSF or calcium-free aCSF. Small drops of 50-200 $\mu \mathrm{m}$ diameter $(0.6-4 \mathrm{nl})$ were injected beneath the surface of the slice by pressure ejection (pulse duration, $20-50 \mathrm{msec}$ ) from a glass micropipette (tip diameter, $\sim 5 \mu \mathrm{m}$ ) using a Picospritzer (General Valve, Fairfield, NJ). Figure $2 C$ shows a photomicrograph of a brainstem slice showing the diameter of an effective drop of GABA (circle) in relation to $R$ and $S$. Picrotoxin $(50 \mu \mathrm{M})$ was applied to the bath with a syringe pump.

Labeling. Some somata of the recorded jaw-closing spindle afferents were filled with Neurobiotin. Depolarizing current pulses (1.0-1.5 nA, 1 $\mathrm{Hz}, 500 \mathrm{msec}$ duration) were passed through the recording electrode for 20-30 min to eject Neurobiotin. At the end of the experiment, the slices were fixed in $4 \%$ paraformaldehyde in $0.1 \mathrm{~m}$ phosphate buffer (PB). Neurobiotin was revealed using standard procedures and the ABC kit (Vector Laboratories, Burlingame, CA).

Immunohistochemistry. In five experiments, animals that have been injected with crystals of DiI ( see above) were perfused, first with saline PB (9\% PBS) and then with a fixative solution containing $2 \%$ paraformaldehyde and $0.1 \%$ glutaraldehyde in $0.1 \mathrm{M}$ PBS. Their brainstem was extracted and immersed in the fixative solution containing, in addition, $30 \%$ sucrose until it sank. It was then sectioned in 80 - to $100-\mu \mathrm{m}$-thick slices on a sliding freezing microtome. In two other experiments, 300$\mu \mathrm{m}$-thick slices were prepared as described above (see section on preparation of slices) and immersed in a fixative solution containing $2 \%$ para- 
formaldehyde and $0.1 \%$ glutaraldehyde in 0.1 м PB for $24 \mathrm{hr}$. In all experiments, the sections were rinsed and incubated with a polyclonal rabbit anti-GABA antibody (1:200 and 1:500; Sigma, St. Louis, MO) or with a polyclonal rabbit anti-glutamate decarboxylase (GAD) antibody (1:1000; Chemicon, Temecula, CA) for $36 \mathrm{hr}$. The primary antibody was revealed with a goat-anti-rabbit secondary antibody conjugated to Alexa 633 or to Alexa 488 (1:100; Molecular Probes). After several rinses, the sections were mounted with GelMount (Biomeda, Foster City, CA) and examined using a multiphoton confocal microscope (excitation wavelengths of 488,633 , and $543 \mathrm{~nm}$ for Alexa Fluor 488, Alexa Fluor 633, and DiI respectively; emission filters: band pass 500-550 for Alexa 488, low pass 650 for Alexa 633, and low pass 560 for DiI when combined with Alexa 488 or band-pass $565-615$ for DiI combined with Alexa 633).

\section{Results}

Close apposition of immunoreactive GABAergic fibers and central axons

We combined immunocytochemistry against GABA and GAD with confocal imaging in an attempt to localize contacts made by GABAergic fibers onto axons of DiI-labeled afferents. In four of four experiments with GABA and three of three experiments with GAD, immunoreactive boutons (Fig. 2D,E, green boutons) were seen in close apposition (white arrows) to large labeled afferent stem axons (Fig. $2 D, E$, red).

\section{Axonal application of GABA impedes propagation of antidromic action potential}

Recordings were made from 77 neurons in NVmes, and 17 cells filled with Neurobiotin were successfully recovered. All had the typical pseudo-unipolar morphology of primary afferent with a single process emerging from a round or ovoid cell body (Fig. 2C, inset). The cells had an average resting membrane potential of $-57 \pm 1 \mathrm{mV}$ and an input resistance of $30 \pm 2 \mathrm{M} \Omega$. Their threshold for firing during current injection was $-44 \pm 1 \mathrm{mV}$, and the amplitude and duration of their spikes were $67 \pm 1 \mathrm{mV}$ and $1.03 \pm 0.03 \mathrm{msec}$, respectively. These spikes were generally followed by a relatively small and short afterhyperpolarization $(9.0 \pm 0.4 \mathrm{mV} ; 5.7 \pm 0.2 \mathrm{msec})$.

Antidromic action potentials were elicited by electrical stimulation of the descending tract at the level of the SupV $(n=57)$, the IntV $(n=11)$, the ventral NVmes $(n=7)$, and the NVmt $(n=6)$. The responses obtained were considered as antidromic on the basis of four criteria. First, they were all-or-none responses that did not appear to arise from an underlying EPSP, and this was confirmed by hyperpolarizing the cell until the spike was abolished. Second, all occurred at very short and constant latencies (mean, $0.63 \pm 0.02 \mathrm{msec}$; minimum, 0.4 and maximum, 1.1 $\mathrm{msec}$ ). Neurons in which stimulation caused spikes with latencies above $1.2 \mathrm{msec}$ or that were of variable latency were not included in the analysis. Third, all responses followed high-frequency (100$250 \mathrm{~Hz}$ ) stimulation without alteration. Finally, they were not affected by removal of $\mathrm{Ca}^{2+}$ from the medium in 12 of 12 cases tested.

GABA was ejected along the axonal tract of 64 cells. In 22 cases (34\%), GABA abruptly abolished the spikes, with no concomitant change in membrane potential in 20 of them (Fig. $3 A$ ). This occurred within seconds and lasted from 0.5 sec to $2 \mathrm{~min}$. A block

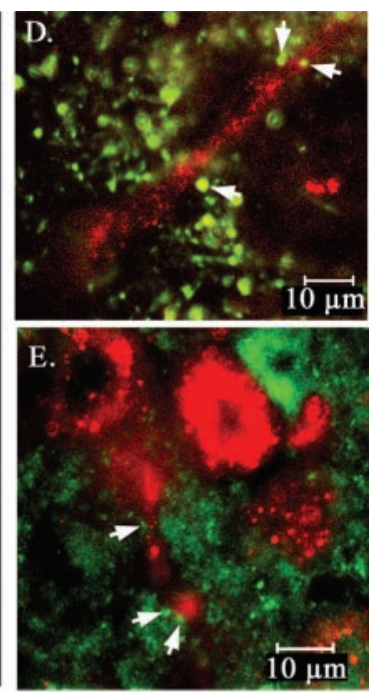

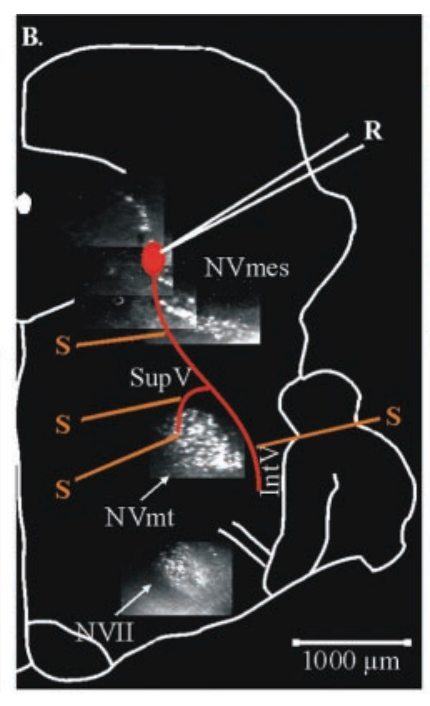

Figure 2. Illustration of the preparation used for recordings. $A$, Sagittal drawing of the brainstem showing a trigeminal muscle 列

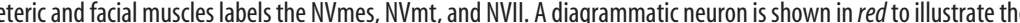
recording and stimulation positions. Inset, The recorded neuron had a single axon emerging from an ovoid cell body. D, E, GABA$(D)$ and GAD- $(E)$ immunoreactive boutons ( green) seen in close apposition (white arrows) to large afferent stem axon (red).

was also produced by pressure ejection of muscimol $(50 \mu \mathrm{M})$, a $\mathrm{GABA}_{\mathrm{A}}$ agonist ( $n=2$; data not shown), and it was reversed by bath application of picrotoxin $(50 \mu \mathrm{M})$, a $\mathrm{GABA}_{\mathrm{A}}$ receptor antagonist $(n=3)$ (Fig. 3A). GABA and muscimol were effective in $\mathrm{Ca}^{2+}$-free, high-magnesium aCSF ( $n=2$ for each), suggesting that they were acting directly on the axon of the recorded neuron.

It has been suggested that presynaptic depolarization of axonal terminals could cause conduction failure at axonal branch points (Henneman et al., 1984), but this is unlikely to be the explanation for our findings. If the applied GABA was causing strong depolarization of the terminals or even of the stem axon (Lamotte et al., 1998), we would have expected it to generate action potentials. Such action potentials would be readily detectable. In fact, GABA and muscimol applied along the axonal tract elicited spontaneous firing in only four cases (Fig. 3B), and there was no concomitant block of the electrically evoked antidromic potentials in the three that were fully tested. This is consistent with the results of Lomeli et al. (2000), who showed that even strong PAD does not cause conduction failure in large stem axons.

In a second group of 16 neurons (25\% of total), GABA applications to the axon did not block the spikes, but it did reduce their amplitude significantly $(-9 \pm 2 \mathrm{mV} ; p<0.001)$ (Fig. 4A). Spike amplitude recovered after washout. In most of these neurons $(n=13)$, there was a simultaneous depolarization of resting somatic membrane potential of $2-10 \mathrm{mV}$. However, in contrast to what was observed with somatic applications of GABA (see below), there was no clear relationship between the magnitude of the depolarization and the decrease in spike amplitude $(r=$ 0.282 ; $p>0.05$ ) (Fig. $4 B$ ), probably because the axonal depolarization occurred at variable distances from the soma and decayed before reaching the intrasomatic recording electrode.

We tried to reproduce the effects of direct GABA application by activation of local circuits that might release GABA under physiological conditions. Conditioning pulses ( $0.1-0.3 \mathrm{msec} \mathrm{du}-$ ration) were delivered to areas that are active during fictive mas- 


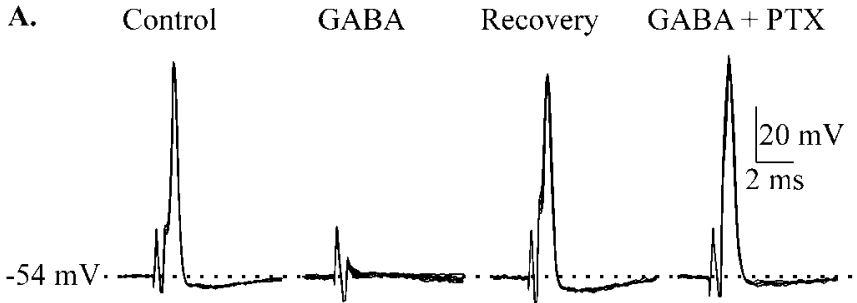

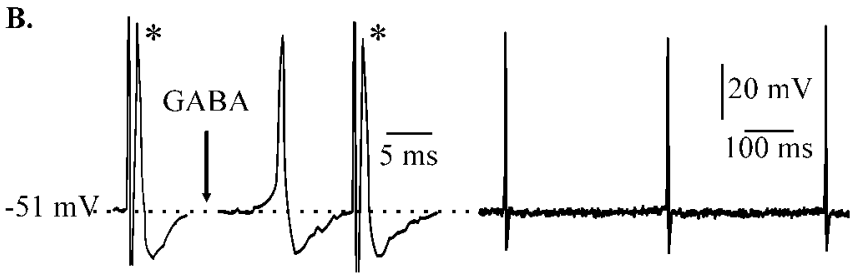

C.

Control

C-T interval $2 \mathrm{~ms}$

C-T

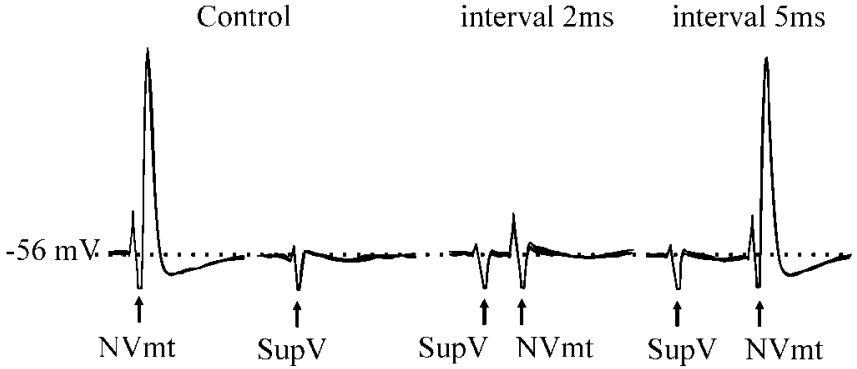

D.

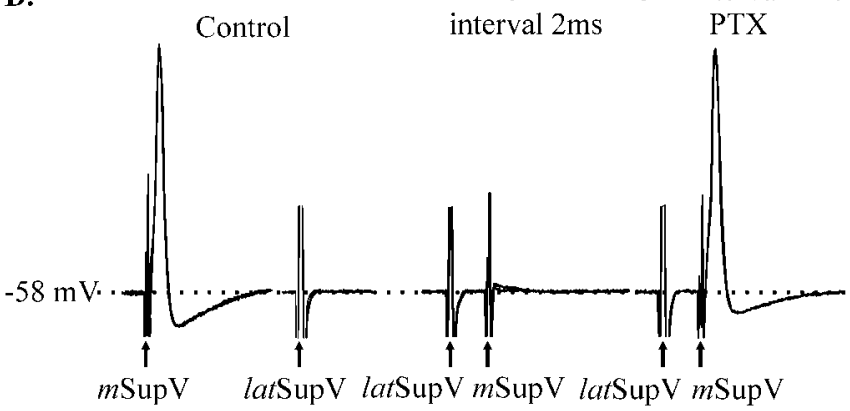

Figure 3. Block of conduction of antidromic action potentials. A, Antidromic potentials elicited by stimulation of the descending tract at the level of IntV (Control) were abolished by pressure application of GABA (1 mM) on the axon (GABA). After recovery (Recovery), bath application of picrotoxin $(50 \mu \mathrm{M})$ prevented the effect of GABA (GABA + PTX). B, Application of GABA (10 mm; arrow) along the axon of another neuron elicited spikes, but this did not stop the propagation of antidromic potentials evoked by electrical stimulation $\left({ }^{*}\right)$. C, Stimulation of the SupV alone had no effect on the soma of a third neuron (Control), but it abolished antidromic spikes caused by stimulation of the NVmt (Control) when the conditioning-test $(C-T)$ interval was $2 \mathrm{msec}$ but not $5 \mathrm{msec}$. D, Blockage of antidromic spikes evoked by stimulation of the medial SupV (mSupV), after stimulation of the lateral SupV (IatSupV), was prevented by bath application of picrotoxin $(50 \mu \mathrm{m})(C-T 2$ msec interval PTX). Stimulation of the lateral SupV by itself had no detectable effect. Calibration bars in $A$ apply to $C$ and $D$. All panels present five superposed traces in $A$ and $C$ and four in $D$.

tication and that are known to contain GABAergic neurons (the intertrigeminal area, the supratrigeminal area, the trigeminal principal sensory nucleus, the parvocellular reticular formation, and the nucleus pontis caudalis) (Li et al., 1996; Turman and Chandler, 1994; Kolta et al., 2000; Bourque and Kolta, 2001).
Stimulation of the dorsal cap of the trigeminal principal sensory nucleus ( 3 of 13) and the adjacent lateral supratrigeminal area (7 of 11) abolished the antidromic spike, but the other sites were ineffective. In eight of these cases, the effect was abrupt and similar to that of axonal GABA applications (as in Fig. 3C), and recovery occurred as soon as the stimulation stopped. The conditioning pulses did not elicit somatic potentials (Fig. 3C, second set of traces from left), suggesting that its effect was exerted at a site remote from the soma. In most cases, a conditioning-test interval of $2 \mathrm{msec}$ corresponding to a monosynaptic latency (Bourque and Kolta, 2001) was effective, but longer intervals were usually not (Fig. $3 C$ ). In two cells, bath application of picrotoxin $(50 \mu \mathrm{M})$ reversed the effect of the trigeminal principal sensory nucleus stimulation and the lateral supratrigeminal area stimulation, indicating that the spikes were blocked via $\mathrm{GABA}_{\mathrm{A}}$ receptors. In the other two cells, stimulation reduced spike amplitude and was accompanied by changes in kinetics like those produce by somatic GABA applications (see below).

\section{Somatic applications of GABA produce different effects from axonal applications}

GABA was applied to the soma of 21 neurons to ensure that the effects of axonal applications were not attributable to diffusion to the cell bodies. GABA depolarized the cells $(2-17 \mathrm{mV}$ in 19 of 21 cells) and decreased their input resistance by $9-58 \%$ (in five of five cells). In contrast, no significant changes of somatic input resistance were detected during axonal GABA application $(n=$ 26). Unlike the axonal applications, somatic GABA rarely abolished antidromic spikes ( 2 of 21$)$. Instead, it reduced their amplitude by $24 \pm 4 \mathrm{mV}$ ( 14 of $21 ; p<0.001$ ) (Fig. $4 C$ ) and increased their duration by $0.46 \pm 0.08 \mathrm{msec}$; ( 14 of $21 ; p<0.05$ ). This also contrasts with axonal applications in which reduction in spike amplitude was not associated with changes in duration, latency, rise, or decay times. Furthermore, there was a strong relationship between changes in membrane potential and spike amplitude $(r=0.858 ; p<0.001)$ (Fig. $4 D)$ with somatic applications. These effects were present in $\mathrm{Ca}^{2+}$-free, high- $\mathrm{Mg}^{2+}$ aCSF (two of two cells tested; data not shown). In all cases, the recovery was gradual and was rarely complete until $1 \mathrm{~min}$ after the application.

\section{Discussion}

The transmission of action potentials along the caudal central axon of trigeminal muscle spindle afferents seems to be regulated during mastication (Kolta et al., 1995; Westberg et al., 2000). Here we presented strong evidence that propagation of antidromic spikes in these sensory neurons is controlled by $\mathrm{GABA}_{\mathrm{A}}$ receptors located on the caudal axon. We also showed that stimulation of some of the cell groups that form part of the masticatory central pattern generator (CPG) (Donga and Lund, 1991; Inoue et al., 1992) mimicked this effect. Furthermore, we observed GABAergic boutons in close apposition to labeled spindle afferent axons. It seems clear from our results that the effects of GABA on the axon cannot be explained by spread of GABA to either the presynaptic terminals or the cell body of these afferents.

This is the first description of a chemical mechanism powerful enough to completely block action potential propagation in large axons, although GABA has been shown to modify spikes in crayfish sensory afferents when it is applied near the first major branch point. The effect is a reduction in the amplitude of incoming action potentials through a combination of depolarization and shunting that depends on increased chloride conductance (Cattaert and el Manira, 1999; Cattaert et al., 2001). These effects are likely to be synaptically mediated because GABA-containing 
axonal boutons have been seen close to the branch point (Cattaert and el Manira, 1999). In the case reported here, the blockage of action potentials by GABA applications to the axon is likely to be attributable to a conduction shunt and/or depolarization of the axon sufficient to inactivate $\mathrm{Na}^{+}$channels (Segev, 1990; Graham and Redman, 1994). We could not distinguish between these two mechanisms because we were recording from the soma. However, it seems likely that the complete failure of spikes was caused by local current shunt with little depolarization, because the soma was not depolarized concomitantly in 20 of 22 cells. Depolarization-induced inactivation of axonal $\mathrm{Na}^{+}$channels may have been involved in the cases in which a reduction in the amplitude of the spike was observed, because this was always accompanied by slight depolarization of the soma, but the observation that the degree of depolarization did not correlate with the reduction in spike amplitude suggests that shunting may have also been involved in these cases.

We suggest that modulation of conduction along trigeminal spindle afferent axons during mastication allows two regions of the axon and their respective collaterals to fire independently from each other and to transmit different messages to their postsynaptic targets during one phase of the movement (Fig 1). As a result, the central axon may become a premotoneuron, carrying signals from the CPG via a set of presynaptic terminals to motoneurons, whereas the rostral portion of the neuron provides feedback from its receptors, although even this is phasically gated by the CPG.

Although this is the first direct evidence of a new mechanism that controls action potential traffic in vertebrate neurons, it is probably not unique to the trigeminal sensory system, and its presence may explain the results of others. For instance, Wall and his colleagues showed that action potentials propagate easily along the large ascending branches of dorsal column afferents studied in vitro, but they often failed to penetrate the thin branches that travel caudally. This tonic blockade could be lifted by picrotoxin, a $\mathrm{GABA}_{\mathrm{A}}$ receptor antagonist (Wall, 1994, 1995; Wall and Bennett, 1994; Wall and McMahon, 1994). It is possible that synapses placed at strategic sites on these and other axons could routinely confine the propagation of action potentials to a portion of the axonal tree during movement and perhaps under other circumstances. Bifunctional and even multifunctional neurons may also exist outside the trigeminal sensory system, which may help explain the function of antidromic firing that has been reported to occur in other sensory afferents (Dubuc et al., 1985; Gossard et al., 1991; Cattaert et al., 1994) and in other neural systems (Pinault, 1995).

\section{References}

Aghajanian GK, Rasmussen K (1989) Intracellular studies in the facial nucleus illustrating a simple new method for obtaining viable motoneurons in adult rat brain slices. Synapse 3:331-338.

Bae YC, Nakagawa S, Yasuda K, Yabuta NH, Yoshida A, Pil PK, Moritani M, Chen K, Nagase Y, Takemura M, Shigenaga Y (1996) Electron microscopic observation of synaptic connections of jaw-muscle spindle and periodontal afferent terminals in the trigeminal motor and supratrigeminal nuclei in the cat. J Comp Neurol 374:421-435.

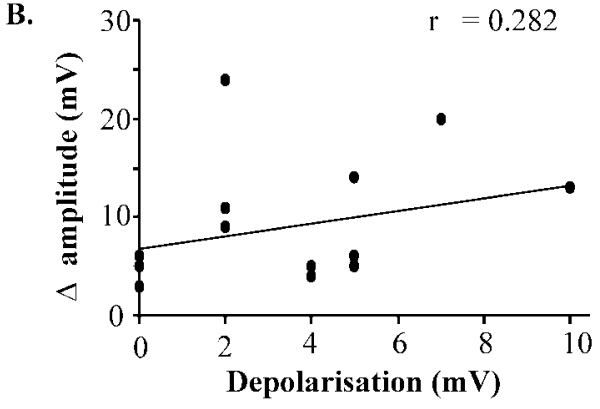

D.

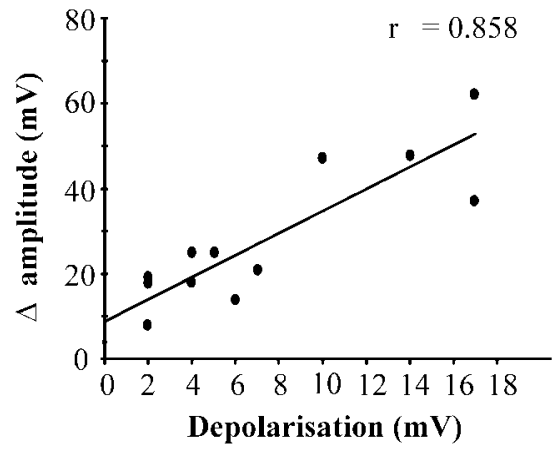
Bevengut M, Clarac F, Cattaert D (1997) Antidromic modulation of a pro-
prioceptor sensory discharge in crayfish. J Neurophysiol 78:1180-1183.

Bourque M-J, Kolta A (2001) Properties and interconnections of trigeminal interneurons of the lateral pontine reticular formation in the rat. J Neurophysiol 86:2583-2596.

Cattaert D, el Manira A (1999) Shunting versus inactivation: analysis of presynaptic inhibitory mechanisms in primary afferents of the crayfish. J Neurosci 19:6079-6089.

Cattaert D, el Manira A, Clarac F (1992) Direct evidence for presynaptic inhibitory mechanisms in crayfish sensory afferents. J Neurophysiol 67:610-624.

Cattaert D, el Manira A, Clarac F (1994) Chloride conductance produces both presynaptic inhibition and antidromic spikes in primary afferents. Brain Res 666:109-112.

Cattaert D, Libersat F, el Manira A (2001) Presynaptic inhibition and antidromic spikes in primary afferents of the crayfish: a computational and experimental analysis. J Neurosci 21:1007-1021.

Dessem D, Taylor A (1989) Morphology of jaw-muscle spindle afferents in the rat. J Comp Neurol 282:389-403.

Donga R, Lund JP (1991) Discharge patterns of trigeminal commissural last-order interneurons during fictive mastication in the rabbit. J Neurophysiol 66:1564-1578.

Dubuc R, Cabelguen J-M, Rossignol S (1985) Rhythmic antidromic discharges of single primary afferents recorded in cut dorsal root filaments during locomotion in the cat. Brain Res 359:375-378.

Eguibar JR, Quevedo J, Jiménez I, Rudomin P (1994) Selective cortical control of information flow through different intraspinal collaterals of the same muscle afferent fiber. Brain Res 643:328-333.

Eguibar JR, Quevedo J, Rudomin P (1997) Selective cortical and segmental control of primary afferent depolarisation of single muscle afferents in the cat spinal cord. Exp Brain Res 113:411-430.

Gossard JP (1996) Control of transmission in muscle group 1A afferents during fictive locomotion in the cat. J Neurophysiol 76:4104-4112.

Gossard JP, Cabelguen J-M, Rossignol S (1991) An intracellular study of muscle primary afferents during fictive locomotion in the cat. J Neurophysiol 65:914-926. 
Gossard JP, Bouyer L, Rossignol S (1999) The effects of antidromic discharges on orthodromic firing of primary afferents in the cat. Brain Res 825:132-145.

Graham B, Redman S (1994) A simulation of action potentials in synaptic boutons during presynaptic inhibition. J Neurophysiol 71:538-549.

Henneman E, Luscher HR, Mathis J (1984) Simultaneously active and inactive synapses of single Ia fibres on cat spinal motoneurones. J Physiol (Lond) 352:147-161.

Inoue T, Masuda Y, Nagashima T, Yoshikawa K, Morimoto T (1992) Properties of rhythmically active reticular neurons around the trigeminal motor nucleus during fictive mastication in the rat. Neurosci Res 14:275-294.

Kolta A, Lund JP, Westberg KG, Clavelou P (1995) Do muscle spindle afferents act like interneurons during mastication? Trends Neurosci 18:441.

Kolta A, Westberg KG, Lund JP (2000) Identification of brainstem interneurons projecting to the trigeminal motor nucleus and adjacent structures in the rabbit. J Chem Neuroanat 19:175-195.

Lamotte dB, Meunier C, Monnet ML, Jami L, Zytnicki D (1998) Reduction of presynaptic action potentials by PAD: model and experimental study. J Comput Neurosci 5:141-156.

Lazarov NE (2002) Comparative analysis of the chemical neuroanatomy of the mammalian trigeminal ganglion and mesencephalic trigeminal nucleus. Prog Neurobiol 66:19-59.

Li YQ, Takada M, Kaneko T, Mizuno N (1996) Gabaergic and glycinergic neurons projecting to the trigeminal motor nucleus: a double labeling study in the rat. J Comp Neurol 373:498-510.

Lomeli J, Quevedo J, Linares P, Rudomin P (1998) Local control of information flow in segmental and ascending collaterals of single afferents. Nature 395:600-604.

Lomeli J, Castillo L, Linares P, Rudomin P (2000) Effects of PAD on con- duction of action potentials within segmental and ascending branches of single muscle afferents in the cat spinal cord. Exp Brain Res 135:204-214.

Luo P, Li J (1991) Monosynaptic connections between neurons of trigeminal mesencephalic nucleus and jaw-closing motoneurons in the rat: an intracellular horseradish peroxidase labeling study. Brain Res 559:267-275.

Pinault D (1995) Backpropagation of action potentials generated at ectopic axonal loci: hypothesis that axon terminals integrate local environmental signals. Brain Res Rev 21:42-92.

Segev I (1990) Computer study of presynaptic inhibition controlling the spread of action potentials into axonal terminals. J Neurophysiol 63:987-998.

Turman Jr JE, Chandler SH (1994) Immunohistochemical evidence for GABA and glycine-containing trigeminal premotoneurons in the guinea pig. Synapse 18:7-20.

Wall PD (1994) Control of impulse conduction in long range branches of afferents by increases and decreases of primary afferent depolarisation in the rat. Eur J Neurosci 6:1136-1142.

Wall PD (1995) Do nerve impulses penetrate terminal arborizations? A prepresynaptic control mechanism. Trends Neurosci 18:99-103.

Wall PD, Bennett DLH (1994) Postsynaptic effects of long-range afferents in distant segments caudal to their entry point in rat spinal cord under the influence of picrotoxin or strychnine. J Neurophysiol 72:2703-2713.

Wall PD, McMahon SB (1994) Long range afferents in rat spinal cord. III. Failure of impulse transmission in axons and relief of the failure after rhizotomy of dorsal roots. Philos Trans $\mathrm{R}$ Soc Lond B Biol Sci 343:211-223.

Westberg KG, Kolta A, Clavelou P, Sandstrom G, Lund JP (2000) Evidence for functional compartmentalization of trigeminal muscle spindle afferents during fictive mastication in the rabbit. Eur J Neurosci 12:1145-1154. 